\title{
Value Networks and Two-Sided Markets of Internet Content Delivery
}

\author{
Nan Zhang a, ${ }^{\text {, }}$, Tapio Levä ${ }^{\text {a }}$, Heikki Hämmäinen ${ }^{\text {a }}$ \\ ${ }^{a}$ Department of Communications and Networking, Aalto University, Otakaari 5, 02150 \\ Espoo, Finland, \{nan.zhang, tapio.leva, heikki.hammainen\}@aalto.fi \\ * Corresponding author. Tel.: +358504355846
}

Accepted manuscript. Please cite this article as:

Nan Zhang, Tapio Levä, Heikki Hämmäinen (In Press). Value Networks and Two-Sided Markets of Internet Content Delivery. Telecommunications Policy, Available online 16 May 2013, ISSN 0308-5961, http://dx.doi.org/10.1016/j.telpol.2013.03.004.

\begin{abstract}
:
The volume and importance of content is increasing in the Internet, whereas the ability of the Internet architecture to scale to the growing demand for transport capacity is uncertain. Even though the natural growth in the demand continues, the growth in traffic volumes can be limited by reducing unnecessary content copying and redundant transportation of the same content. Information-centric networking (ICN), featuring globally unique naming of content and optimized in-network caching, has been suggested as a potential future solution to significantly reduce unnecessary traffic, but its economic feasibility has not been widely studied. This paper evaluates the economic feasibility of ICN by using the two-sided markets theory to analyze four Internet content delivery models: the client-server model, content delivery network (CDN) model, peerto-peer model and ICN model. Value networks and two-sided markets of these content delivery models are identified in the process. The results suggest that content providers can be willing to pay for the lower delay of content delivery in ICN, if ICN can solve its coordination problems related to cost-allocation, contracting, quality of service guarantees, and content usage statistics. These incentive challenges are essentially the same as in-network web caching originally faced and could not overcome. Internet
\end{abstract}


access providers may also consider investing in the deployment of ICN due to reduced interconnection costs. However, the ICN model may require a revenue-creating business model to make it more attractive to Internet access providers than the CDN model that provides similar cost savings.

Keywords: two-sided markets, Internet content, value network, information-centric networking, content delivery network, peer-to-peer

\section{Introduction}

Fundamental changes are taking place in the Internet as the traffic and content volumes keep growing. By 2016, 86\% of global consumer traffic is estimated to be video (Cisco, 2012) and a significant part of video content is cacheable by nature (e.g. Cha, Kwak, Rodriguez, Ahn, \& Moon, 2007; Zink, Suh, Gu \& Kurose, 2009), which indicates that the importance of caching is increasing. A concrete example of the impact of caching comes from Uganda and Kenya, where the introduction of Google Global Cache increased users' Internet traffic volume by $300-1000 \%$ in only two weeks (Kende, 2012). Notably, content sources are consolidating and most Internet inter-domain traffic already today flows directly between large content providers (CPs), content delivery networks (CDNs) and consumer networks (Labovitz, Iekel-Johnson, McPherson, Oberheide \& Jahanian, 2010). As a consequence, the demand is shifting from plain (peer-to-peer) connectivity to value-added (video) content delivery services.

The original end-to-end Internet architecture has not been able to solve the scalability and coordination problems of quality and caching in content delivery, which has led to the fragmentation of the Internet to overlay networks, such as CDNs (Clark et al., 2006). The growth of the CDN market is challenging the traditional transit and peering models, creating the need for a more uniform and efficient content delivery model better integrated to the Internet architecture. A new networking paradigm, information-centric networking (ICN), utilizes in-network caching and adapts the network architecture to the content-driven network usage patterns (Ahlgren, Dannewitz,

Imbrenda, Kutscher, \& Ohlman, 2012). The concept introduces routing based on unique content names instead of content location (Dannewitz, 2009; Jacobson et al., 2012). In addition to the U.S. based Named Data Networking (NDN) project, two EU-funded 
research projects, SAIL and PURSUIT, have studied the concept. Even though the technical challenges are driving the research, the importance of the socio-economic aspects and stakeholder incentives should not be underestimated.

Studying the market dynamics of a technology just being defined is not simple. Our approach is to analyze similar technologies existing in the market and then draw conclusions based on the findings. In content delivery, CDNs utilize distributed caching closer to the end-user and focus on content instead of location, and thus can be considered as a precedent of ICN. In addition, peer-to-peer (P2P) networks distribute content all over the edge of the networks (i.e. in end-users' devices), which is also comparable to in-network caching of ICN.

Interestingly, a CDN can be considered as a two-sided platform, which aims at getting both CPs and Internet access providers (IAPs) on board with incentivecompatible cost allocation. Acting as a platform between CPs and IAPs (i.e. content consumers), CDNs have also been able to solve the coordination problems related to inter-domain quality of service (QoS). Due to the platform role and the network externalities existing in two-sided markets, CDNs have been a successful content delivery model (Faratin, 2007). Therefore, understanding how widely two-sided pricing is used in Internet content delivery is highly relevant in predicting the success of ICN.

In this paper, the feasibility of ICN is investigated based on the analysis of the following current and new Internet content delivery models: the client-server model, CDN model, P2P model and ICN model. Eight expert interviews covering key stakeholders (Internet service providers, CPs and data center providers) in Finland during 2010 (Zhang, 2010) are used as an input for identifying the value networks and two-sided markets of the content delivery models.

The rest of this paper is organized as follows. Section 2 gives a brief overview into the theories and background information underlying this work, such as the two-sided market theory and interconnection pricing. Section 3 describes the value networks of Internet content delivery models, so that the identification and analysis of two-sided markets is possible in Section 4. Section 5 presents a comparison of the different models and discusses the implications of the identified two-sided markets for the feasibility of ICN. Finally, Section 6 concludes the paper. 


\section{Background}

To lay the basis for the analysis, the different Internet content delivery models are discussed in this section. In addition, two-sided pricing and interconnection pricing are presented briefly. Finally, the value network analysis theory and notations are described.

\subsection{Internet Content Delivery Models}

This paper focuses on content delivery between CPs and consumers, and on content delivery models that the CPs can use. The client-server model, CDN model, P2P model and ICN model are alternative solutions for CPs to reach their audience and can exist in parallel. These models are briefly explained here.

\subsubsection{Client-Server Model}

In this paper, the client-server model means the basic end-to-end connection between the $\mathrm{CP}$ and the consumer. In the client-server model, the $\mathrm{CP}$ stores the content in a centralized server or pool of servers. The servers can be owned by the $\mathrm{CP}$ or leased as a service from data centers or cloud providers. The consumers, in this model, request content from these centralized servers based on the address of the servers.

Caching has been deployed in the client-server model in the form of web caches, which are designed to improve the experience of web browsing by lowering latency and reducing traffic volume in the Internet backbone and around the web servers (Huston, 1999). Web caching (Barish \& Obraczka, 2000) can happen either on the client side, where visited web sites are cached in the client's device or in a close-by proxy server for a period of time, or on the server side, where for example search engines can cache search results. However, in this paper, web caching is excluded from the client-server model to differentiate it from the rest of the models.

\subsubsection{Content Delivery Network Model}

The CDN model is an overlay to the basic Internet, which divides the end-to-end connection into two separate connections: one between the $\mathrm{CP}$ and the $\mathrm{CDN}$ provider, the other between the CDN provider and the consumer. The content is stored either permanently or temporarily at the CDN provider's servers, which are located closer to 
the end-users and, thus, reduce latency for end-users. However, Triukose, Wen and Rabinovich (2011) suggest that CDNs may not yet have succeeded to fully exploit the delay cutting potential of decentralized edge caching. The other value added by a CDN is reliability of the network, better throughput and origin server load balancing (Vakali \& Pallis, 2003). Akamai (Dilley et al., 2002), for example, is a large CDN provider that stores the CPs' content in its servers around the world closer to the consumers.

Among the thin literature on economics of CDNs, Hau, Burghardt and Brenner (2011) observe that the use of CDNs and CP multi-homing is likely to improve the pricing power of terminating ISPs due to locked in end-users. Additionally, Faratin (2007) is the first to analyze the CDN as a two-sided market.

\subsubsection{Peer-to-Peer Model}

P2P networks represent another type of overlay to the basic Internet (Schollmeier, 2002), which consist of distributed resources connected by the network. The P2P model has the same network components as the client-server model. The only difference is that the clients connected to the network can also act as servers, whereas in the client-server model, the roles of client and server are distinct. A P2P network can be either controlled, where the end-users are authenticated and authorized, or uncontrolled, where all users can access the network. In this work, the focus is on content provider controlled P2P networks, where the consumers have to authenticate themselves with the $\mathrm{CP}$ before having access to the content.

The Swedish online film service provider Voddler (Sjöman, 2010) is an example of a CP controlled P2P network. The content is permanently stored at Voddler's servers. Once streamed to a consumer, the content is cached temporarily on the consumer's device. From these devices it can be streamed to other consumers to reduce delay, origin server load, and Voddler's distribution costs (Bergström, Davidsson \& Zhou, 2008).

\subsubsection{Information-centric Networking Model}

Routing in the ICN concept is based on what data is sought instead of where the data is (Jacobson et al., 2012). The basic idea of ICN is that the network has cache servers that cache data and the data moves freely in the network. In addition, ICN applies a unique naming scheme to all information objects in the network, where the naming scheme 
offers, for example, data integrity, owner continuity and owner identification (Ahlgren et al., 2012). Naming is also the main difference between ICN and the traditional web caching.

Every node in the network can potentially act as a cache server (Ahlgren et al. 2012). In practice, when a piece of content traverses from the origin server to the host that has expressed interest in the content, the network elements in between cache the content. If other hosts in the same area request the same content, any one of the network elements that sees the request may reply with the content. As a consequence, the importance and load of origin servers diminish. In addition, cache placement and its economic feasibility is an important question, which is widely discussed by, for example, Krishnan, Raz and Shavitt (2000), Chen, Katz and Kubiatowicz (2002), Xu, Li and Lee (2002), Tang and Chanson (2002), Jia, Li, Hu, Wu and Du (2003), Sourlas, Flegkas, Paschos, Katsaros and Tassiulas (2011) and Agyapong and Sirbu (2012).

Four recently proposed ICN architectures are Data Oriented Network Architecture (DONA) (Koponen et al., 2007), Named Data Networking (NDN) (Zhang et al., 2010), the publish/subscribe (pub/sub) paradigm (Fotiou, Trossen \& Polyzos, 2012) and Networking of Information (NetInf) (Ahlgren \& Vercellone, 2010). Though the different ICN architectures share many assumptions and architectural properties, they differ in details. For example, unique naming is a key component of all ICN architectures, but the pub/sub is more dependent on lower level host addresses due to the rendezvous routing scheme, in which publishers and subscribers are matched (Fotiou et al., 2012). For a more detailed comparison of DONA, NDN, pub/sub and NetInf, and their functionalities, refer to Ahlgren et al. (2012).

Similarly to the P2P network, caching in the ICN model can be either controlled or uncontrolled by CPs and other stakeholders. In this work, an ISP controlled transparent caching model is considered, where the ISPs place cache servers in their networks into optimal locations based on user demand and economic factors. This means that no business agreements on caching are assumed to exist between the CPs and ISP. Thus, the content is cached based only on the ISP's preference without other stakeholders' intervention. However, it should be noted that technically ICN has the capability to offer CPs control over their content distribution and caching; for example, the scoping 
concept introduced in pub/sub networks (Trossen \& Kostopoulos, 2012). Thus, the ICN model in this work does not reflect any single ICN architecture, but is based on the common conceptual assumptions of ICN. The ISP controlled transparent caching model is chosen for simplicity, because in an agreement-based model, the CP needs to have agreements with several ISPs in order to have wide coverage for its content (Salo et al., 2011). For an analysis on the agreement-based model, please refer to Levä, Zhang and Flinck (2013).

\subsection{Two-sided Market Theory}

Two-sided market theory belongs to the field of economics called industrial organization, which studies the structure of markets and strategic interactions between firms (Faratin, 2007). The theory explains the behavior of firms in markets that exhibit two-sidedness and is closely related to network externalities (Parker \& Van Alstyne, 2005). Rochet and Tirole (2006) define two-sided markets as markets with two distinct sides that are interlinked (through a platform) and where not only the overall price level matters, but also the price structure between the two sides.

The demand asymmetries between interlinked markets typically lead to skewed pricing with one side charged more than the other, which is shown in Fig. 1. In Fig. 1, $\mathrm{p} 1$ and $\mathrm{p} 2$ illustrate the normal pricing, where both sides are priced separately regardless of the other side, and q1 and q2 mark the respective quantities demanded. The checked areas represent the revenues gained from both sides. The skewed two-sided pricing is shown with p1' and p2', where side 1's price is lowered and side 2's raised. As a consequence of side 1's lower price, side 1's quantity demanded increases to q1'. Due to cross-side network externalities, the increased demand on one side increases the demand also on the other side, which is represented with a demand curve shifting to the right (D2 $\rightarrow$ D2'). This in turn causes side 2's quantity demanded to increase as well as the possibility for side 2 to raise its prices to $\mathrm{p} 2$ '. The resulting combined revenue for the platform from both sides is bigger than in the normal pricing case and is shown with the solid areas. For example, Caillaud and Jullien (2003), Eisenmann, Parker and Van Alstyne (2006), Faratin and Wilkening (2006), Hagiu and Yoffie (2009), and Genakos and Valletti (2011) illustrate the skewed pricing applied in real world cases. 
Side 1 demand

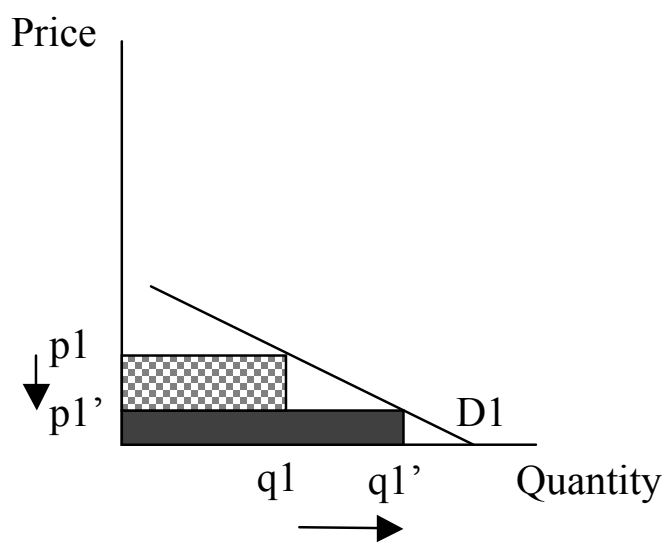

Side 2 demand

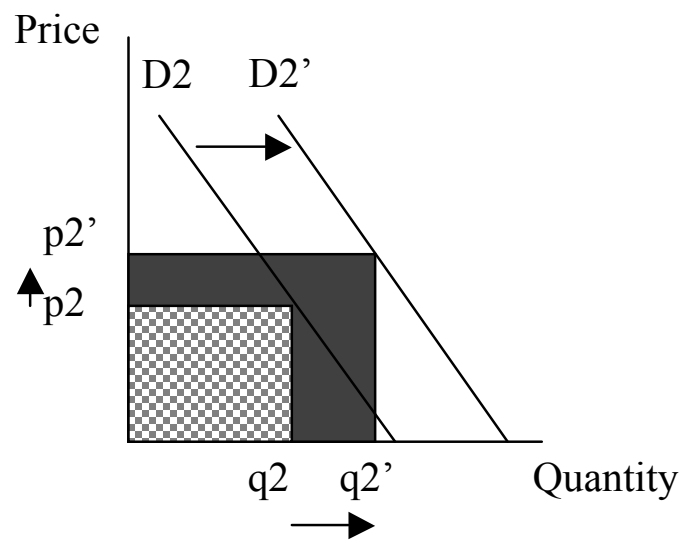

Fig. 1. Skewed pricing.

Usually, the side that brings more value to the platform or has relatively more elastic demand is subsidized (Armstrong, 2006). Subsidizing in two-sided market terminology is defined as charging the one side less than the other side, which often leads to pricing the more valuable side under production costs. The previously mentioned Voddler, for example, employs two-sided pricing in its business model. 80\% of the content Voddler distributes is free for consumers (Sjöman, 2010), because consumers are more price sensitive than the advertisers on the other side of the market. Advertisers pay Voddler for showing a few minutes of advertising before each viewing.

Though two-sided markets are mostly identified for single-homing cases, in reality, multi-homing happens on both sides of the platform, where players connect with more than one platform. For example, Caillaud and Jullien (2003), Gabszewicz and Wauthy (2004), Rochet and Tirole (2006), Armstrong (2006), Choi (2010), and Hau, Burghardt and Brenner (2011) all propose their own models of a two-sided market with multihoming either on one side or both sides. They conclude that when multi-homing happens on only one side, the single-homing side has the potential for monopolistic pricing. At the same time, the multi-homing content providers are reaching more endusers, but this reduces the exclusivity of the platform. In this work, the effect of multihoming is discussed only briefly, because the focus is on comparison of the different 
Internet content delivery models rather than building extensive two-sided market models.

\subsection{Interconnection Pricing}

Since the two-sided markets are formed through asymmetric cost-allocation, the pricing models of Internet connectivity form the basis of the analysis. This paper assumes that the consumer access pricing is flat-rated, though IAPs increasingly adopt usage caps (BEREC, 2012; Van der Veen, 2011). In addition, content providers are assumed to be charged based on the transit pricing model discussed later in this section. Moreover, to simplify the analysis in this work, the current Internet interconnection models (i.e., transit and peering contracts) are taken as given also in the future, even though realization of ICN may require completely different cost-allocation mechanisms. In fact, new types of agreements, such as partial transit and paid peering (Clark et al., 2008), have already emerged into Internet interconnection as a consequence of the increased complexity of the Internet architecture. In addition, researchers and policy makers are investigating the possibility to include a content value component in the interconnection agreements, as against to interconnection agreements for only traffic volume (Clark, Lehr \& Bauer, 2011).

Peering is a bilateral agreement between two operators to access each other's customers (Norton, 2011a). No monetary compensations are paid by either of the operators. Peering agreements are not transitive, which means that the operators can only access each other's customers but not the rest of the network. On the other hand, operators in transit agreement buy access to the upstream operator's whole network (Norton, 2011a). This means that transit agreements provide access to all the nodes in the upstream operator's routing table and it is the upstream operator's responsibility to provide connectivity to the whole Internet for the lower level operators. Charging in transit agreement is often based on the bigger volume of either the inbound or outbound traffic and realized using 95th percentile billing principle (Norton, 2011a).

Understanding the difference between the on-net and off-net traffic is also important, because they have different marginal costs for an IAP. Traffic is said to be on-net, if both end-points are located in the same IAP's network; and off-net, if the endpoints are located in different IAPs' networks. As a consequence, on-net traffic is 
cheaper for an IAP to deliver. Off-net traffic can be delivered either through peering or transit agreements, where peering typically is the cheaper option for an IAP.

\subsection{Value Network Analysis}

Value network analysis gives a solid foundation for the two-sided market analysis, because it shows graphically the stakeholders and the value exchanges between them. Value chains (Porter, 1985) and value networks (Stabell \& Fjeldstad, 1998) have been widely used in many studies, but Allee (2000a) is the first one to divide the value exchanges between stakeholders into different categories. Allee's Three Currencies of Value include 1) goods, services and revenue, 2) knowledge, and 3) intangible benefits. The first currency includes the actual goods or services and monetary payments flowing between stakeholders. Knowledge in this context means the possession of strategic information, planning and process knowledge and employee competence, such as technical know-how. Customer loyalty, sense of community, and image enhancement are examples of intangible benefits as outlined by Allee (2000b, 2008).

In addition, in his work on mobile Internet, Zhao (2008) adopts a modified categorization of value exchanges to better fit the networking context. These are services and goods, monetary benefit, and intangible benefits. In Zhao's categorization, knowledge is included in the intangible benefits as a type of information instead of as its own currency.

The value network notation used in this work follows the basic idea of Allee's (2000a) configuration with a few modifications based on Zhao's (2008) configuration and on Faratin's (2007) notation to better suit the context of this study. The currency names used are thus: traffic/content transfer, monetary transfer and intangible benefits. Recognizing the intangible benefits is valuable, especially in the networking context, because they together with two-sided pricing provide reasons why certain goods or services can be given for free (i.e. without monetary compensation). Thus, the intangible benefits are further divided into three categories based on Allee's (2000b, 2008) definition of intangibles: brand recognition, information and loyalty. 


\section{Value Networks of Internet Content Delivery}

The stakeholders and value networks of each Internet content delivery model were identified in eight expert interviews conducted in Finland during spring 2010. The semistructured interviews lasted 45-90 minutes and the interviewees covered three key stakeholders: Internet service providers (ISPs), CPs and data center providers. The discussion revolved around the features of content delivery models, stakeholder costs and revenues, and current market structure and market evolution prospects of Internet content delivery. In addition, academic experts were interviewed for a better understanding of the current Internet architecture as well as the ICN functionalities. Due to the lack of significant $\mathrm{CDN}$ providers in Finland, no $\mathrm{CDN}$ providers were interviewed. However, one of the ISPs also provides CDN services and one of the CPs is using CDN services, which helped in improving the understanding of CDNs. For the list of interview questions and complete result summary, please refer to Zhang (2010).

Several stakeholders affecting the formation of the value network were identified from the interviews; the ones considered in this value network analysis are defined as follows. Content producer produce the content, content providers act as portals for aggregated content and consumers (Cs) use the content. Data center providers supply server space, whereas Internet service providers provide Internet connectivity and include Internet access providers and Internet backbone providers (IBPs). IAPs operate access networks and IBPs backbone networks. In addition, CDN providers offer CDN services, advertisers insert advertisements into final products in the distribution process and sponsors input their brand names during the content making process.

The Internet content delivery activities can be divided into two layers: the content service layer, where the product is digital content like video clips or pieces of music, and the Internet interconnection layer, where the product is transportation of content over the Internet. In the content service layer, the level of monetary transfer depends on the value of the content and can vary between different pieces of content, whereas in the Internet interconnection layer, the level of monetary transfer reflects the bits transferred and is indifferent to the content.

Even though we focus on the Internet interconnection layer, Fig. 2 shows the simplified value network of the content service layer, which stays the same regardless 
of the underlying Internet content delivery model. The different value exchanges in the value network are denoted with different line styles and arrow heads: traffic transfer of the content with solid line, monetary compensation with dashed line, and transfer of intangible benefits with dotted line. Fig. 2 can also be considered as the CP's internal value network as it shows the origin of the content, the different ways the content can be stored and the different revenue sources of the CP.

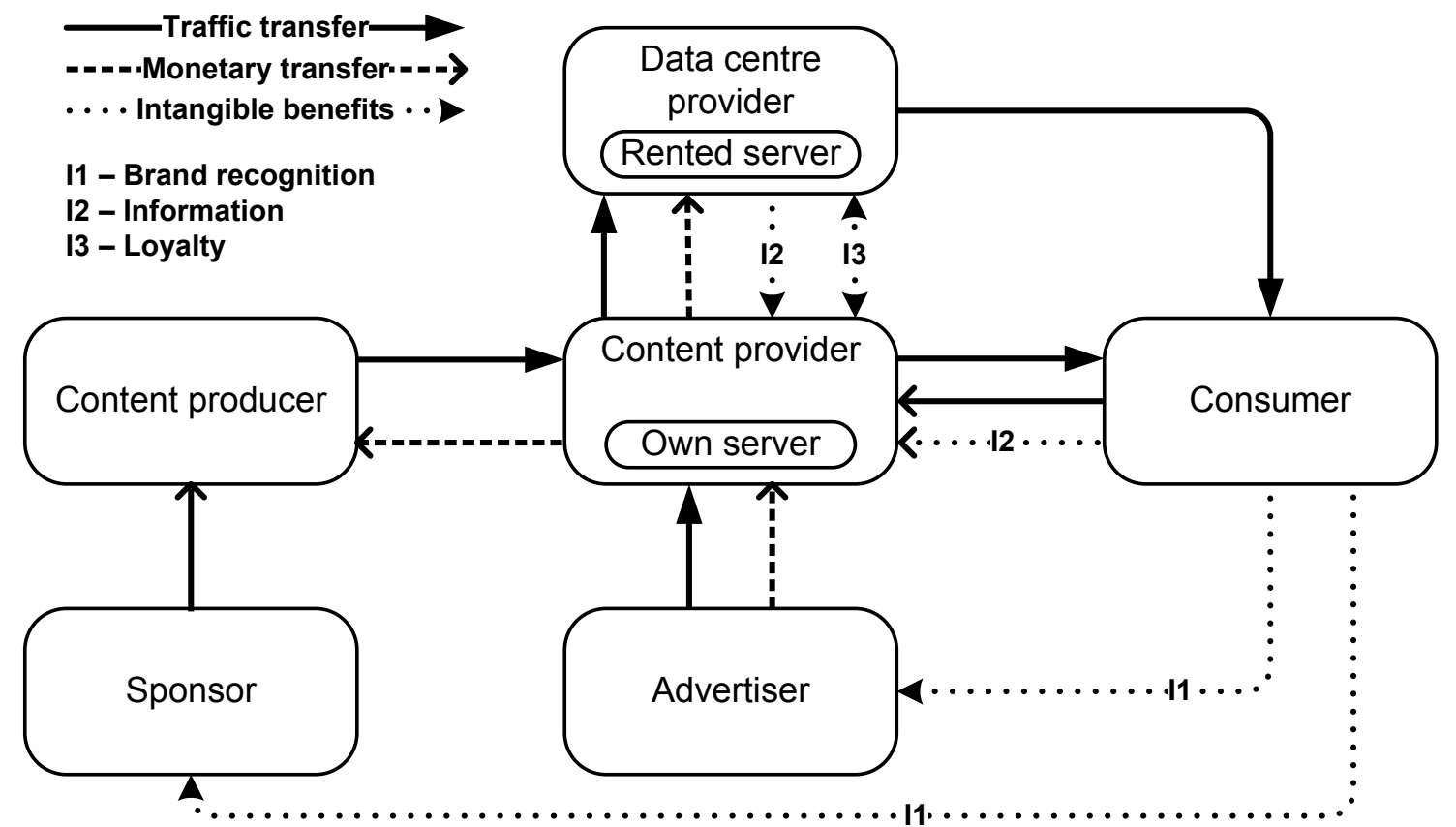

Fig. 2. Simplified value network of the content service layer.

Fig. 3 presents the value networks of the Internet interconnection layer for each of the four content delivery models: a) client-server model, b) CDN model, c) P2P model and d) ICN model. The value network notation follows the notation of Fig. 2. The traffic transfer arrows show only the direction of the content traffic, not the direction of content requests, which flow in the opposite direction. Additionally, the relative traffic and monetary volume between stakeholders is presented with the difference in thickness of the arrows, and the relative sizes of same stakeholder groups are shown with size difference between the stakeholder boxes (e.g. IAP1 vs. IAP2). The client-server model (Fig. 3a) is chosen as the base case, and the differences to that model are highlighted by using darker arrows in subfigures b-d. 


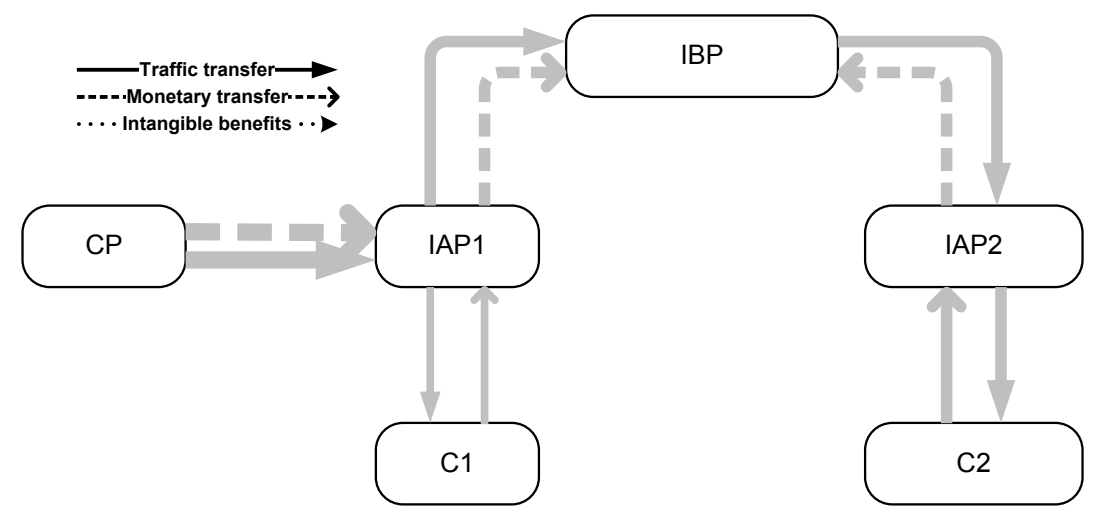

a) Value network of the client-server model.

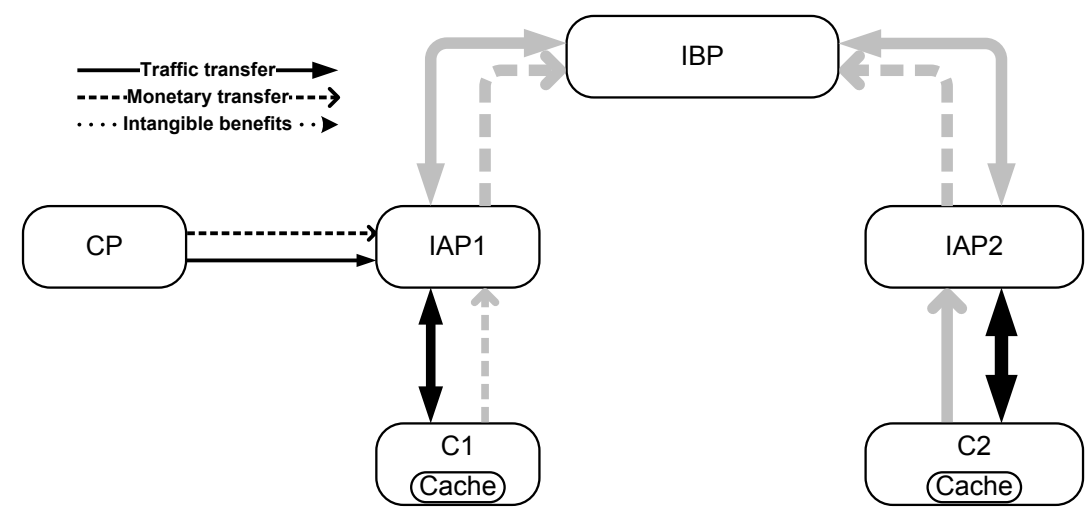

c) Value network of peer-to-peer model.

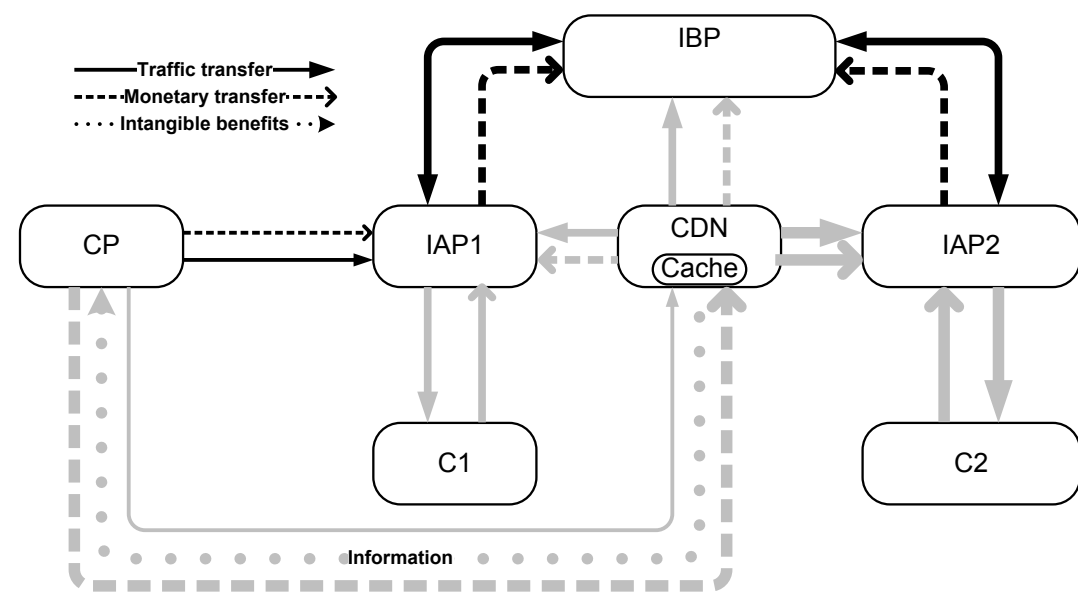

b) Value network of the CDN model.

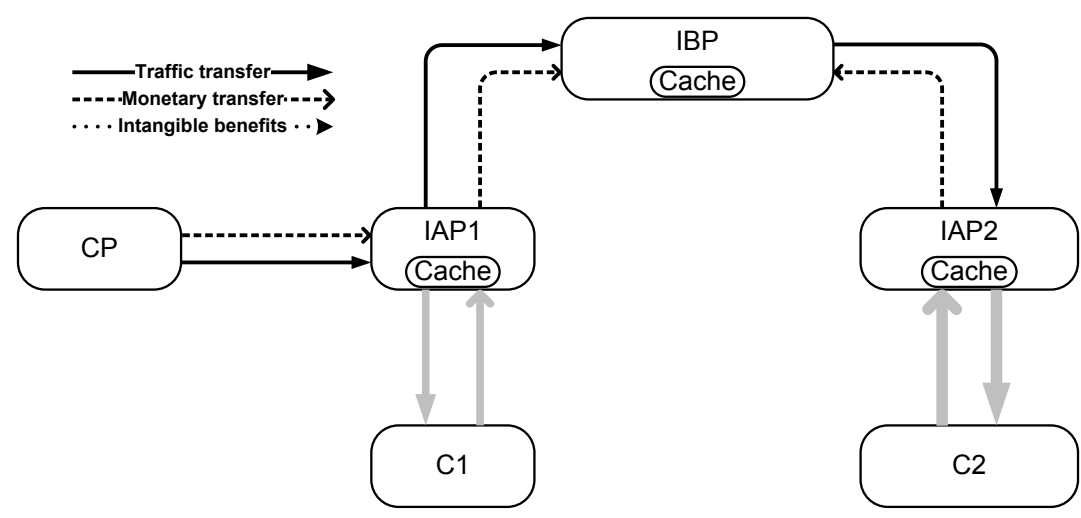

d) Value network of information-centric networking model.

Fig. 3. Value networks of four content delivery models: a) client-server model, b) CDN model, c) peer-to-peer model, and d) information centric networking model. 


\subsection{Value Network of Client-Server Model}

In the client-server model (Fig. 3a), all content requests are served from the CP's servers through its access provider IAP1, which is shown with the thick traffic arrow between CP and IAP1. It should be noted that the CP can also connect directly to the IBP, but for simplicity, the connection is left out from the models in this paper since adding it would not change the findings. Consumers in the consumer group 1 (C1) have the same IAP (IAP1) as the CP or are reachable through the IAP1's peering links. Consumer group 2 (C2), on the other hand, includes the rest of the users not included in C1, whom the CP can reach only through IBP. Due to this division, C2 is assumed to be bigger in size than $\mathrm{C} 1$. As a consequence, IAP2 is also bigger than IAP1 and the traffic between the consumers and their respective access provider is of different volumes. The traffic into the IBP always has the same volume as the outbound traffic from the IBP, because IBP presents all the Tier-1 providers, through which the traffic traverses. In addition, the IBP only has inward monetary transfers as all the other stakeholders connected to it have to pay for the connection.

For the $\mathrm{CP}$, the location of consumers $(\mathrm{C} 1 \& \mathrm{C} 2)$ is irrelevant from the pure transport cost perspective, because its monetary transfer to IAP1 equals the traffic transfer from CP to IAP1. However, from the perspective of end-user experience, consumers reachable only through IBP often suffer from significant latencies due to longer distance from the CP and possibly more limited bandwidth, which may affect the popularity of the CP's content service among those consumers. For the IAPs, the location of consumers is more important as it defines the IAPs' monetary transfers (i.e., transit costs) to IBP. The more consumers belong to $\mathrm{C} 2$, the larger are the costs of IAP1 and IAP2, and the revenue of the IBP.

\subsection{Value Network of CDN Model}

In the CDN Model (Fig. 3b), the CP outsources (at least part of ${ }^{1}$ ) the content delivery to a $\mathrm{CDN}$ provider, which is added to the figure as a new stakeholder. The $\mathrm{CP}$ can be

\footnotetext{
${ }^{1}$ Typically, the delivery of heavy, popular and delay-sensitive content, such as video traffic, is served by CDNs.
} 
connected to the CDN either through the IAP1 or directly through a peering link as is shown in the figure. Now the content requests are served from the CDN's servers. As a result, the traffic volume between the CP and IAP1 decreases. Nevertheless, this does not necessarily reduce the CP's costs as it pays the CDN for the volume of traffic delivered to end-users. Thus, the monetary transfer from the CP to the CDN equals the traffic transfer between the CDN and IAPs rather than the traffic transfer between the $\mathrm{CP}$ and the CDN. For example, Norton (2011b) compared four video distribution models (Internet transit, CDN, hybrid of transit and peering, and P2P) with prices from 2006 and concluded that the CDN model is the most costly for the CP, especially with low traffic volumes. On the other hand, the CDN may charge less for the traffic than an IAP does when the traffic volume is large enough. For example, the transit price for 2012 is estimated to be 2.34 USD per Mbps (Norton, 2011a) and a content provider with 400Mbps a month may pay as low as 2 USD per Mbps (Rayburn, 2012) to the CDN provider. However, CDN prices depend heavily on the traffic volume delivered, ranging from 2 USD per Mbps to 11 USD per Mbps (Rayburn, 2012), and, thus, are not illustrated in the constructed value network.

In case the cost differences between CDN and client-server models are small, the CP's incentive to use CDN stems from the other benefits that the CDN provides: namely, better scalability, decreased load on CP's origin server and improved end-user experience especially to $\mathrm{C} 2$. The $\mathrm{CDN}$ is also able to collect valuable usage information, which is shown as an intangible benefit in the figure.

In the emergence of the CDN model, IAP1 loses part of its revenues received from the $\mathrm{CP}$, but also saves in the transit costs (as does IAP2) since the traffic is delivered to consumers over the peering links between the CDN and the IAPs. Traditionally, CDNs and IAPs had settlement-free peering agreements (Norton, 2011a) and IAPs allowed CDNs to collocate servers in the IAPs networks for free. However, due to the increase of heavy traffic going through the CDN, IAPs increasingly charge CDNs for peering (Clark et al., 2011). In Fig. 3b, the fee charged by the IAP from the CDN corresponds to the traffic volume going through the CDN and the IAPs. However, in reality, the terms of paid peering agreements vary and may not depend strictly on the traffic volume, which causes conflicts between the CDN providers and the IAPs (Level3, 2010). 
Finally, from the IBP's perspective, the CDN provider is just another customer of its transit service. However, the CDN provider competes for the IBP's content delivery market and decreases the IBP's revenue. The negative impact of the CDN model depends on the number of peering agreements between the CDN and the IAPs. If the coverage of the CDN's peering is extensive, less traffic and money is transferred between IBP and IAP1/IAP2/CDN.

\subsection{Value Network of P2P model}

In the P2P model (Fig. 3c), the CP controls an exclusive P2P network consisting of customers in consumer groups $\mathrm{C} 1$ and $\mathrm{C}^{2}$ as in the case of Voddler, for example. Content is cached in and the content requests are served from the consumers' devices instead of CP's own servers or the CDN. As a result, compared to the client-server model, the outbound traffic volume from the CP decreases similarly as in the CDN model. More interestingly, and contrary to the CDN model, also the transport costs of the CP decrease significantly as they are outsourced to IAPs.

The CP has no cost incentive to optimize from where the content requests are served; thus, the P2P network is not location aware ${ }^{3}$ (Karagiannis, Rodriguez \& Papagiannaki, 2005). As a result, the requests by $\mathrm{C} 1$ may be served from $\mathrm{C} 2$, and vice versa, which leads to an increase in traffic volumes between the IAPs and their consumers. Thus, the decrease in the CP's transport costs is accomplished by moving most of the distribution costs to the IAPs, which, despite the increased traffic in the access network, receive the same amount of payments from consumers due to consumers' flat rate pricing (Rodriguez, Tan \& Gkantsidis, 2006). On the other hand, the traffic volumes between the IAPs and the IBP stay the same as in the client-server model. This arrangement, however, is not sustainable for IAPs. As a result, IAPs are

${ }^{2}$ The CP could also rely on a more general purpose P2P network, such as BitTorrent, but due to content control problems leading to copyright infringements and challenges in charging, this paper focuses on the controlled model.

${ }^{3}$ The CP may still have incentive to serve content from nearby caches due to service quality reasons, which often has positive impact on the transit costs of the IAPs. 
increasingly adopting traffic management schemes to throttle P2P traffic or set usage caps (BEREC, 2012). For example, AT\&T implemented a tiered usage cap scheme in 2011, where a 150 GB monthly data cap was put on all of its DSL customers and a 250 GB cap on its U-Verse users (Bode, 2011).

In addition, even though the P2P model is more cost-efficient for the $\mathrm{CP}$ than the client-server model or the CDN model, it may not be suitable for every CP. Building the required components and controlling a $\mathrm{P} 2 \mathrm{P}$ network requires expertise that many CPs do not possess. The model is also very application-specific, where traffic volume is limited by the adoption of a certain software or application, for example, Voddler Player (Voddler, 2013).

\subsection{Value Network of ICN model}

Though several value networks can be constructed for ICN (Salo et al., 2011), this paper concentrates on a value network, where IAP transparently caches named content similarly to web caching. The ICN model presented in Fig. 3d resembles the P2P model, but now the caches are located at and controlled by the ISPs. The CP loses the overall control on the content delivery as the ICN system transparently caches the content and serves the content requests from the optimal, nearby locations. As a result, traffic and monetary transfers between the CP and the IAP1 decrease to the same level as in the CDN and P2P models.

Due to the optimal cache selection, also the traffic volume through the Internet backbone decreases similarly as in the CDN model. Therefore, the monetary transfer between the IAPs and the IBP reduces, which may lead to resistance from the IBPs and lower their interest to participate in the ICN deployment. The increased costs for the IAP from building and maintaining the cache infrastructure are not illustrated in the value network, because only traffic related costs are modeled. However, these costs are taken into consideration in the discussions later in the paper.

\section{Two-sided Markets in Internet Content Delivery}

This section identifies and analyzes the most important two-sided markets in both the content service and Internet interconnection layers based on both prior literature and the 
value networks constructed in Section 3. However, because the focus of this paper is on the different content delivery models and not on the services provided, the content service layer's two-sided markets will be discussed only briefly before looking into the Internet interconnection layer's two-sided markets in more detail.

\subsection{Identified Two-sided Markets}

The identified two-sided markets are shown in Table 1, where side 1 shows the subsidized side. The advertising, sponsorship and content sharing markets are located in the content service layer, whereas the other two-sided markets are located in the Internet interconnection layer.

Table 1. Two-sided markets in Internet content delivery.

\begin{tabular}{|c|c|c|c|c|}
\hline Layer & Platform & $\begin{array}{l}\text { Side } 1 \\
\text { (subsidized } \\
\text { side) }\end{array}$ & Side 2 & Market Name \\
\hline \multirow{3}{*}{ 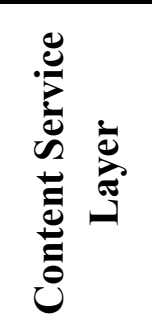 } & $\begin{array}{l}\text { Content } \\
\text { provider }\end{array}$ & Consumers & Advertisers & $\begin{array}{l}\text { Advertising } \\
\text { Market }\end{array}$ \\
\hline & $\begin{array}{l}\text { Content } \\
\text { producer }\end{array}$ & Consumers & Sponsors & $\begin{array}{l}\text { Sponsorship } \\
\text { Market }\end{array}$ \\
\hline & $\begin{array}{l}\text { Content } \\
\text { provider }\end{array}$ & Consumers & $\begin{array}{l}\text { Content } \\
\text { producers }\end{array}$ & $\begin{array}{l}\text { Content Sharing } \\
\text { Market }\end{array}$ \\
\hline \multirow{3}{*}{ 总 } & $\begin{array}{l}\text { Internet access } \\
\text { provider }\end{array}$ & Consumers & $\begin{array}{l}\text { Content } \\
\text { providers }\end{array}$ & IAP Market \\
\hline & $\begin{array}{l}\text { Internet } \\
\text { backbone } \\
\text { provider }\end{array}$ & Eyeball-IAPs & Content-IAPs & IBP Market \\
\hline & CDN provider & ISPs & $\begin{array}{l}\text { Content } \\
\text { providers }\end{array}$ & CDN Market \\
\hline
\end{tabular}

The content service layer's two-sided markets have already been discussed in detail in previous studies. For example, Hagiu and Yoffie (2009) discuss the advertising market's two-sidedness with a Google case and Rochet and Tirole (2003) explain the sponsorship and content sharing markets through content production and streamingmedia technology cases. 
From the Internet interconnection layer's two-sided markets, the IAP and IBP markets exist in all four content delivery models. In the CDN model, a unique two-sided market - the CDN market - is present, of which a similar analysis has been done earlier by Faratin (2007). However, in the ICN model, no significant two-sidedness (as defined by the two-sided market theory) is found, the consequences of which are discussed in Section 5.2 together with the challenges of ICN in general. Though the two-sided markets may exist simultaneously in several of the content delivery models, each market is discussed here separately for clarity.

\subsection{IAP Market}

In the IAP market, the IAP (platform) connects consumers and CPs, where consumers are individual end-users in the Internet and CPs provide commercial content. The subsidy side in this two-sided market is not fully clear and depends on the price elasticity of demand of the two sides. Generally, the consumers' demand is assumed to be more price elastic than the CP's demand and, thus, the consumer side is assumed to be subsidized. This point is further illustrated by the commonly used flat rate pricing for consumers (Odlyzko, 2000). The CP's price elasticity of demand depends on whether multi-homing is practiced.

A single-homing CP tends to perceive an IAP with the most consumers as offering the most value. Thus, this IAP attracts more CPs and due to network effect, even more consumers will join the network. As a consequence and due to potential consumer lockin schemes by the IAP, the CPs' demand for this IAP's service is less price elastic and the IAP can set higher prices for the CPs. In addition, when CPs gain more consumers and possibly profit, more content is created for distribution and more CPs may enter the market, which in turn may attract more consumers. This cycle will continue until the market is saturated. The IAPs in this case can grow into regional monopolies.

In reality, multi-homing is very common for CPs, which diminishes the market power of a single IAP. Thus, the CP's demand is more price elastic in the multi-homing case. In addition, network effect is weaker with $\mathrm{CP}$ multi-homing and the two-sided platform tends to grow slower than in the single-homing case. 
On the other hand, IAPs are interconnected and the CPs connected to IAP1 are also reachable by the consumers connected to IAP2. Thus, consumers do not need to choose IAPs based on the availability of content and this may suggest that the IAP market is not a true two-sided market. However, the incentive for two-sided pricing may come from end-users' quality of experience (QoE). If both the consumer and the $\mathrm{CP}$ are connected to the same IAP, the QoE may be better than for the consumers connected to another IAP. Thus, the consumers prefer an IAP with more CPs and vice versa.

\subsection{IBP Market}

The Internet backbone provider is the platform in the IBP market, with "eyeball" heavy IAPs (IAP1) and "content" heavy IAPs (IAP2) on the two sides. Eyeball heavy IAPs (eyeball-IAPs), such as Verizon or AT\&T, connect consumers to the Internet, whereas content heavy IAPs (content-IAPs), such as Abovenet, host a lot of content servers (Clark et al., 2008). In the Internet content delivery context, eyeball-IAPs are expected to generate mainly outbound traffic while content-IAPs produce inbound traffic to the IBP.

The two-sidedness of the market is based on the cost asymmetries of the inbound and outbound traffic, where the IBP incurs more costs from the terminating IAP (i.e. eyeball-IAP) due to hot-potato routing, in which the originating IBP has an incentive to pass on the off-net traffic as soon as possible to the terminating IBP (Laffont, Marcus, Rey \& Tirole, 2003; Clark et al., 2011). On the other hand, the eyeball-IAP and the content-IAP are assumed to be charged with the same transit unit price despite the eyeball-IAP causing more costs to the IBP (Norton, 2011a). Thus, the eyeball-IAP is being subsidized ${ }^{4}$ in the IBP market. Though the bigger volume from the inbound or outbound traffic is billed, it does not affect the analysis, because in a single-homing situation, the outbound traffic from a content-IAP should equal the inbound traffic towards an eyeball-IAP. However, with one side multi-homing with several IBPs, the

${ }^{4}$ The definition of subsidy does not usually include the benefits arising from cost asymmetries. But for consistency within this paper, the eyeball-IAP is considered as being subsidized. 
multi-homed side produces more traffic than the single-homing side and this might affect the two-sidedness of the market.

The subsidy to eyeball-IAPs is passed on to the consumers, who perceive more value from the connection and, thus, may generate more traffic for both the eyeball-IAP and IBP. As a consequence, the CPs connected to the content-IAP may gain more clients or more advertising revenue, which may lead to more profit. However, when the eyeball-IAP's off-net traffic increases, it faces higher costs and lower profits. If the costs of eyeball-IAPs rise too high, they might want to limit the volume of off-net traffic or establish more peering agreements with other IAPs. In addition, eyeball-IAPs may also raise prices for all consumers to cover the increasing costs and declining profit. Alternatively, if the eyeball-IAPs are not able to pass on the higher costs onto consumers, the revenue may not cover costs and they may go out of business.

Following the same logic as in the IAP market, the increase in both content supply and consumers increases the demand for Internet connectivity services. As a conclusion, the two-sided pricing in this two-sided market is beneficial for the IBPs as the higher Internet penetration likely increases their revenue. For the IAPs in general, and the eyeball-IAPs charging flat prices from consumers in particular, the situation may be the opposite as the off-net traffic increases and IAPs may face the potential threat of going out of business.

\subsection{CDN Market}

The CDN model presents its own two-sided market with the CDN provider as the platform. The two sides are CPs and IAPs. When CDNs first emerged into the market, big CDN providers tested two business models: Akamai charged CPs and Inktomi charged ISPs for the service (Faratin \& Wilkening, 2006). Seeing that Akamai's revenue model now dominates the $\mathrm{CDN}$ market, charging the CPs for the service rather than ISPs seems to be more feasible. This is because the IAPs are more price elastic than the CPs, who are more willing to pay for better-than-best-effort services. Thus, as can be seen from Fig. 3b, the IAPs are creating costs for the CDN provider instead of revenue; in other words, ISPs are being subsidized in the CDN market. 
When a CDN provider subsidizes IAPs, it will be connected to more IAPs, and thereby has a better reach to the consumers. The CDN with good reach of consumers, generally, is more attractive to CPs. As a consequence, both more CPs and more IAPs connect to this $\mathrm{CDN}$, which then may grow into a monopoly or at least a few large CDNs may start to dominate the market leading to an oligopoly. All stakeholders benefit from this situation, including the consumers who experience better user experience.

On the other hand, multi-homing can happen on both sides of the market in the CDN market: both IAPs and CPs are often connected to more than one CDN provider. This would reduce the positive network effect received by the CDN providers and prevent a single CDN monopoly from forming. In addition, the CDN platform itself may interconnect with other CDNs, as suggested by the IETF's CDNi initiative (Peterson \& Davie, 2012). This would reduce the network effect experienced by the two sides similarly to when IAPs interconnect with each other in the IAP market.

\section{Discussion}

Based on the constructed value networks and identified two-sided markets of the four content delivery models, a comparison of them can be made. In addition, this section discusses the challenges faced by the ICN model.

\subsection{Comparison of Internet Content Delivery Models}

Based on the interview results and previous studies, several parameters have been identified as important to the different stakeholders. In this section, the different content delivery models will be compared using these parameters and a summary is shown in Table 2. The comparison is done with the scale high/medium/low relative to each model and the grey scale represents how preferable (the darker the better) each model is, when evaluated with each parameter. In addition, the comparison is done only on a qualitative level, because the quantification of each parameter is not objective and depends on the perspective of the reader.

The values for the first two parameters (marked with a *-sign in Table 2) are derived from the value network analysis conducted in Section 3 and the values for the 
second set of parameters (marked with a ${ }^{\S}$-sign) are based on prior knowledge on each of the technical models as well as the interview results. Furthermore, the values have their foundation in the assumptions made in Sections 2.1 and 2.3. With a different set of assumptions, the values may be entirely different.

Table 2. Comparison of different Internet content delivery models based on several parameters. The values of parameters marked with $(*)$ are derived from the value network analysis and parameters with $\left({ }^{\S}\right)$ from the technical knowledge and interviews.

\begin{tabular}{|l|c|c|c|c|}
\hline Parameter & $\begin{array}{c}\text { Client-Server } \\
\text { Model }\end{array}$ & CDN Model & P2P Model & ICN Model \\
\hline Cost for IAP* & high & low & high & low \\
\hline Cost for CP* & high & high & low & low \\
\hline $\begin{array}{l}\text { CP's control } \\
\text { over caching }\end{array}$ & high & high & low & low \\
\hline $\begin{array}{l}\text { CP's control } \\
\text { over content } \\
\text { distribution }\end{array}$ & high & high & high & low \\
\hline $\begin{array}{l}\text { Level of } \\
\text { guaranteed } \\
\text { QoS }\end{array}$ & medium & high & low & low \\
\hline $\begin{array}{l}\text { Latency for } \\
\text { end-user }\end{array}$ & high & low & medium & low \\
\hline $\begin{array}{l}\text { Scalability } \\
\text { (low }\end{array}$ & medium & high & high \\
\hline
\end{tabular}

The CDN and ICN models decrease transit costs for IAPs due to CDN and cache servers being closer to the consumers; thus, IAPs may favor these two models. As the cost for IAP values are purely derived from the value network analysis, the increased costs from cache investments in the ICN model are not taken into consideration. The implications of the additional costs are, however, discussed as a challenge in Section 5.2. In addition, the real peering dispute cases between ISPs and CDNs (Level3, 2010; Van der Veen, 2011) show that the IAPs' costs in the CDN model may be higher than the assumptions in this paper suggest. The cost for an IAP in the P2P model can also be low, 
if the recent development towards a locality aware P2P becomes reality (Seedorf \& Burger, 2009). From the IBPs' perspective, reduction in transit traffic decreases its revenue; thus, the client-server and P2P models may be more profitable for IBPs.

From the CP's point of view, P2P and ICN models lower the costs of content delivery. On the other hand, due to the distributed nature of the ICN model, CP's control over its content distribution, such as copyright protection and access control, is low compared to the other models. However, in the ICN model, if the access control information is embedded into the information objects, CPs can have better control over their content.

On a related matter, CP's control over caching (i.e. where to cache, what to cache and how often content is updated in caches) is highest in the CDN model due to the business agreements and in the client-server model due to CP's origin server ownership. The CP's control over caching is low in the transparent caching ICN model, because the CPs and ISPs do not have caching related business agreements. In the P2P model, the CP can have high control over content distribution, but less control over content caching, because the CP cannot control the caching policies of each end-user device.

In addition, providing a certain level of service quality to end-users is important for a $\mathrm{CP}$, and the $\mathrm{CDN}$ model best implements guaranteed service quality. Thus, the lower costs for CPs in other models may not compensate for the possibly lower service quality and control. In addition, the QoS level is low in the ICN model, because of uncontrolled transparent caching, though the different ICN implementations may offer different levels of QoS.

On the other hand, from the end-user's perspective, the CDN and ICN models are preferable due to their low latency. The P2P model may have low latency, depending on from where the content is served. However, the P2P network is assumed to not be location aware. In addition, IAPs are increasingly introducing a tiered pricing scheme to end-users, because the increase of P2P traffic increases the uplink traffic significantly; thus, the end-users may perceive the P2P model to offer less value than the other models. 
From the whole network's view, each model's impact on the Internet scalability is very important. Due to the distributed nature of the network, P2P and ICN models have the highest scalability, which is preferable for all the stakeholders.

\subsection{Challenges of Information-Centric Networking}

Several challenges for the deployment of ICN have been identified based on the key findings of this paper and are discussed in this section. First is the two-sided pricing in the CDN market, which causes CDN providers to consolidate and become Internet wide CDNs. A network wide CDN or interconnected CDN providers (i.e. $\mathrm{CDNi}$ ) would have the same coverage as the ICN model and, thus, can be a direct competitor to ICN. In addition, a CDN provider may move towards ICN by placing more cache servers into the network and closer to the end-users.

The CDN model, being a two-sided market, has a central platform that coordinates the service provisioning and offers service level guarantees. In addition, the CDN provider offers usage statistics to the CPs as a service. The proposed value network model for ICN does not have a unique two-sided market with a central platform that can offer guaranteed service levels and value added services. As CPs value both service level guarantees and usage statistics, the ICN model needs to solve these issues to gain acceptance from CPs. In addition, for the advertisers, who are a revenue source for CPs, being able to identify each individual user is important, but caching makes the identification less straightforward. To solve this problem, IAPs could collect usage and user information for the CPs. However, ICN's ability and IAP's willingness to provide such services is uncertain.

The CP's control over its content is another issue in ICN, especially with dynamic contents such as targeted marketing. This means that conflicts may arise between CPs and IAPs: IAPs may not have the incentive to update content caches very frequently while CPs may wish the content to be up to date. Legal measures, business agreements and monetary incentives may help in solving this conflict and the other limitations of the ICN model identified earlier. Nevertheless, these limitations have to be solved before ICN can prosper. 
The central platform also makes charging of the service simpler. In the ICN model, it is unclear who can monetize the service offering and who should be charged for the service. The CDN market's analysis suggests that CPs, compared to the ISPs, have higher willingness to pay for better-than-best-effort content delivery. Despite this, ISPs' willingness to deploy ICN is more crucial as they control the network locations, in where cache servers should be located. One potential platform could, thus, be the ISPs, if they can pass the extra costs to the consumers or CPs. For example, the name resolution system (i.e., rendezvous) in NetInf and pub/sub can be considered as a twosided market, which matches the requesters to the publishers (Trossen \& Kostopoulos, 2012). A natural owner of this function is the ISP. However, it remains unclear how an ISP could monetize the name resolution and, thus, this two-sided market is not discussed in this paper.

IAPs' willingness to invest in ICN can be determined by summing up the net benefit of reduced off-net traffic, extra investments and other costs. However, the ICN model considered in this paper may not be able to compete with the CDN model, in which the IAPs avoid additional investments but still enjoy the same transit cost savings accompanied by possible payments from CDN providers in case of paid peering. Thus, the IAPs may need further incentives to invest in ICN from possible additional revenues and monetization schemes. For example, Agyapong and Sirbu (2012) argue that an eyeball-IAP has the incentive to invest in caching, if it can charge high enough prices from the CPs. On the other hand, ICN may enable shift towards more content-oriented interconnection agreements, where not only the transferred data volume but also the availability of the content from the caches of a peering partner is taken into consideration (Levä et al., 2013). If the value of the available content is higher than its transfer costs, this new way of peering may serve as additional incentive for the IAPs to invest in ICN.

Finally, based on the value network analysis, IBP's willingness to add cache servers to its network is very low, but it may be stimulated by the significantly falling Internet transit prices (Norton, 2011a). Since transit revenue has traditionally been the major income for IBPs, IBPs are now shifting to other revenue sources such as providing CDN services (Labovitz et al., 2010). Thus, IBPs may also be open to the possibility of finding viable revenue models from ICN. 


\section{Conclusion}

Information-centric networking (ICN), employing unique content naming and optimized caching, is a prominent concept for improving the scalability of the Internet, but it still faces technical and, especially, business challenges. This paper constructed value networks for four content delivery models and performed a two-sided market analysis on each of them: 1) the client-server model without web caching, 2) the content delivery network (CDN) model, 3) the content provider (CP) controlled peer-to-peer (P2P) model and 4) the Internet service provider (ISP) controlled transparent caching ICN model. Based on the value networks and identified two-sided markets, the models were compared using both economic and technical parameters, and the challenges for ICN were identified.

The results suggest that CDNs, a kind of early ICN approach, prosper because they have reduced the coordination problem of end-to-end quality of service (QoS) and have utilized the demand asymmetry between Internet access providers (IAPs) and CPs. Successful deployment of two-sided pricing has made CDNs attractive to both IAPs and CPs and has caused the CDN providers to consolidate and become stronger. The current ICN proposals are missing an incentive model for solving the coordination challenges related to the cost-allocation, contracting, guaranteeing QoS, and content usage statistics, though technically the different ICN architectures partly have these capabilities. An interesting future research topic is whether an incentive model, two-sided or not, can be devised to enable the success of ICN.

The standardized global deployment of ICN requires that the incentives of stakeholders are aligned. Importantly, CPs, as decision drivers, seem to be willing to pay for lower delay in content delivery. However, ISPs' willingness to deploy ICN is even more crucial as they control the network locations, in where the cache servers should be placed. In-network caching reduces IAP's off-net traffic costs, which can motivate IAPs to invest in ICN. However, in addition to cost savings, no revenue model exists yet to attract IAP investments. This may lead to IAPs considering moving into the CDN business, which offers similar cost savings and a revenue model that has been proven to work. Therefore, possible revenue models and value network configurations of ICN need to be studied in the future. 


\section{Acknowledgements}

The authors thank all the interviewed experts for the valuable input data to this work as well as Ph.D. (Tech.) Timo Smura for his comments. This work has been partially funded by the European Commission, under grant FP7-ICT-2009-5-257448-SAIL.

\section{References}

Agyapong, P., \& Sirbu, M. (2012). Economic incentives in information-centric networking: implications for protocol design and public policy. Communications Magazine, IEEE, 50(12), pp. 18-26.

Ahlgren, B. \& Vercellone, V. (2010). Networking of information - an information-centric approach to the network of the future. In: Proceedings of ETSI Future Network Technologies Workshop. Sophia Antipolis, France, 2010, March 10-11.

Ahlgren, B., Dannewitz, C., Imbrenda, C., Kutscher, D. \& Ohlman, B. (2012). A survey of informationcentric networking. IEEE Communications Magazine, 50(7), pp. 26-36.

Allee, V. (2000a). Reconfiguring the value network. Journal of Business Strategy, 21(4), pp. 36-39.

Allee, V. (2000b). The value evolution: addressing larger implications of an intellectual capital and intangibles perspective. Journal of Intellectual Capital, 1(1), pp. 17-32.

Allee, V. (2008). Value network analysis and value conversion of tangible and intangible assets. Journal of Intellectual Capital, 9(1), pp. 5-24.

Armstrong, M. (2006). Competition in two-sided markets. The RAND Journal of Economics, 37(3), pp. 668-691.

Barish, G. \& Obraczka, K. (2000). World Wide Web caching: Trends and techniques. IEEE Communications Magazine, 38(5), pp. 178-184.

BEREC. (2012). BEREC findings on traffic management practices in Europe. Retrieved from http://berec.europa.eu/eng/document_register/subject_matter/berec/reports/45-berec-findings-ontraffic-management-practices-in-europe.

Bergström, M., Davidsson, H. \& Zhou, Y. (2008). U.S. Patent No. 2008/0059631. Alexandria, VA, U.S.

Bode, K. (2011). Exclusive: AT\&T to impose caps, overages. Retrieved from http://www.dslreports.com/shownews/Exclusive-ATT-Will-Soon-Impose-150GB-DSL-CapOverages-113149.

Caillaud, B. \& Jullien, B. (2003). Chicken \& egg: Competition among intermediation service providers. The RAND Journal of Economics, 34(2), pp. 309-328.

Cha, M., Kwak, H., Rodriguez, P., Ahn, Y. \& Moon, S. (2007). I tube, you tube, everybody tubes: Analyzing the world's largest user generated content video system. In: Proceedings of the 7th ACM SIGCOMM conference on Internet measurement, pp. 1-13. 
Chen, Y., Katz, R. \& Kubiatowicz, J. (2002). Dynamic replica placement for scalable content delivery. In: Proceedings of 1st International Workshop on Peer-to-Peer Systems (IPTPS'02). Cambridge, MA, USA.

Choi, J. (2010). Tying in two-sided markets with multi-homing. The Journal of Industrial Economics, 58(3), pp. 607-626.

Cisco. (2012). Cisco visual networking index: forecast and methodology, 2011-2016. (Updated 2012 May Retrieved

from http://www.cisco.com/en/US/solutions/collateral/ns341/ns525/ns537/ns705/ns827/white_paper_c11481360.pdf.

Clark, D., Faratin, P., Gilmore, P., Bauer, S., Berger A., \& Lehr W. (2008). The growing complexity of Internet interconnection. Communications \& Strategies, 72(4), pp. 51-71.

Clark, D., Lehr, W., Bauer, S., Faratin, P., Sami, R., \& Wroclawski, J. (2006). Overlay Networks and the Future of the Internet. Communications and Strategies, 63(63), 109-129.

Clark, D., Lehr, W. \& Bauer, S. (2011). Interconnection in the Internet: the policy challenge. In: Proceedings of 39th Research Conference on Communication, Information and Internet Policy (TPRC '11). Arlington, VA, U.S., 2011 September 23-25.

Dannewitz, C. (2009). NetInf: An information-centric design for the future Internet. In: Proceedings of $3 r d$ GI/ITG KuVS Workshop on the Future Internet.

Dilley, J., Maggs, B., Parikh, J., Prokop, H., Sitaraman, R. \& Weihl, B. (2002). Globally distributed content delivery. IEEE Internet Computing, 6(5), pp. 50-58.

Eisenmann, T., Parker, G., \& Van Alstyne, M.W. (2006). Strategies for two-sided markets. Harvard Business Review, 84(10), pp. 92-101.

Faratin, P. (2007). Economics of overlay networks: an industrial organization perspective on network economics. In: Proceedings of the Joint Workshop on the Economics of Networked Systems and Incentive-Based Computing (NetEcon $+I B C$ ), in conjunction with ACM Conference on Electronic Commerce (EC'07). San Diego, CA, U.S., 2007 June 11.

Faratin, P. \& Wilkening, T. (2006). Interconnection discrimination: a two-sided markets perspective. In: Proceedings of 5th Hot Topics in Networks (HotNets-V'06). Irvine, CA, U.S., 2006 November 2930.

Fotiou, N., Trossen, D., \& Polyzos, G. (2012). Illustrating a publish-subscribe Internet architecture. Telecommunication Systems, 51(4), pp. 233-245.

Gabszewicz, J. \& Wauthy, X. (2004). Two-sided markets and price competition with multi-homing. CORE Discussion Paper No. 2004/30.

Genakos, C. \& Valletti, T. (2011). Testing the "waterbed" effect in mobile telephony. Journal of the European Economic Association, 9(6), pp. 1114-1142.

Hagiu, A. \& Yoffie, D. (2009). What's your Google strategy. Harvard Business Review, April, 2009, pp. 74-81.

Hau, T., Burghardt, D. \& Brenner, W. (2011). Multihoming, content delivery networks, and the market for Internet connectivity. Telecommunications Policy, 35(6), pp. 532-542. 
Huston, G. (1999). Web caching. The Internet Protocol Journal, 2(3). Retrieved from http://www.cisco.com/web/about/ac123/ac147/ac174/ac199/about_cisco_ipj_archive_article09186a0 0800c8903.html.

Jacobson, V., Smetters, D. K., Thornton, J. D., Plass, M. F., Briggs, N. H. \& Braynard, R. L. (2012). Networking named content. Communications of the ACM, 55(1), pp. 117-124.

Jia, X., Li, D., Hu, X., Wu, W. \& Du, D. (2003). Placement of web-server proxies with consideration of read and update operations on the Internet. The Computer Journal, 46(1), pp. 1-14.

Karagiannis, T., Rodriguez, P. \& Papagiannaki, K. (2005). Should internet service providers fear peerassisted content distribution?. In: Proceedings of the 5th ACM SIGCOMM conference on Internet Measurement (IMC '05). USENIX Association, Berkeley, CA, USA.

Kende, M. (2012). Overview of recent changes in the international IP interconnection ecosystem. Presentation at ITU Regional Seminar on Costs and Tariffs for Member Countries of the Regional Group for Asia and Oceania (SG3RG-AO), Bali, Indonesia, 28-30 May 2012. Retrieved from http://www.itu.int/ITU-D/finance/work-cost-tariffs/events/tariff-seminars/Indonesia-

12/pdf/Session5_Kende_IXP.pdf.

Koponen, T., Chawla, M., Chun, B.-G., Ermolinskiy, A., Kim, K. H., Shenker, S. \& Stoica, I. (2007). A data-oriented (and beyond) network architecture. In: Proceedings of the 2007 conference on Applications, technologies, architectures, and protocols for computer communications (SIGCOMM '07). ACM, New York, NY, USA, pp. 181-192.

Krishnan, P., Raz, D. \& Shavitt, Y. (2000). The cache location problem. IEEE/ACM Transactions on Networking (TON), 8(5), pp. 568-582.

Labovitz, C., Iekel-Johnson, S., McPherson, D., Oberheide, J. \& Jahanian, F. (2010). Internet interdomain traffic. In: Proceedings of the 2010 conference on Applications, technologies, architectures, and protocols for computer communications (SIGCOMM'10), pp. 75-86. New Delhi, India.

Laffont, J.-J., Marcus, S., Rey, P. \& Tirole, J. (2003). Internet interconnection and the off-net-cost pricing principle. The RAND Journal of Economics, 34(2), pp. 370-390.

Level3. (2010). News archive: Level3 releases statement to clarify issues in Comcast/Level3 interconnection dispute. Retrieved from http://level3.mediaroom.com/index.php?s=23600\&item $=65047$.

Levä, T., Zhang, N. \& Flinck, H., 2013. Business models of information-centric networking: Adoption strategy for ISPs. Unpublished manuscript submitted to Future Network \& Mobile Summit 2013, Lisbon, Portugal, 2013 July 3-5.

Norton, W.B. (2011a). The Internet peering playbook: Connecting to the core of the Internet. DrPeering Press.

Norton, W.B. (2011b). Video Internet: The next wave of massive disruption to the U.S. peering ecosystem, (v1.7). Retrieved from http://drpeering.net/white-papers/Video-Internet-The-Next-WaveOf-Massive-Disruption-To-The-U.S.-Peering-Ecosystem.html.

Odlyzko, A. (2000). Should flat-rate Internet pricing continue. IT Professionals, 2(5), pp. 48-51. 
Parker, G. G. \& Van Alstyne, M. W. (2005). Two-sided network effects: a theory of information product design. Management Science, 51(10), pp. 1494-1504.

Peterson, L. \& Davie, B. (2012). Framework for CDN Interconnection: draft-ietf-cdni-framework-01. Retrieved from tools.ietf.org/html/draft-ietf-cdni-framework-01.

Porter, M. E. (1985). Competitive Advantage: Creating and Sustaining Superior Performance. Free Press, New York, NY.

Rayburn, D. (2012). CDN pricing stable: Survey data shows pricing down 15\% this year. Retrieved from http://blog.streamingmedia.com/the_business_of_online_vi/2012/09/cdn-pricing-stable-survey-datashows-pricing-down-15-this-year.html.

Rochet, J.-C. \& Tirole, J. (2003). Platform competition in two-sided markets. Journal of European Economic Association, 1(4), pp. 990-1029.

Rochet, J. \& Tirole, J. (2006). Two-sided markets: a progress report. The RAND Journal of Economics, 37(3), pp. 645-667.

Rodriguez, P., Tan, S.-M. \& Gkantsidis, C. (2006). On the feasibility of commercial, legal P2P content distribution. ACM SIGCOMM Computer Communication Review, 36(1).

Salo, J. (Ed.), Biraghi, A.M., Gonçalves, J., Levä, T., Ferreira, R. J., Zhang, N. ... Ohlman, B. (2011). SAIL project deliverable D.A.7: New Business Models and business dynamics of the future networks. Retrieved from http://www.sail-project.eu/wp-content/uploads/2011/08/SAIL_DA7-FinalVersion_public.pdf.

Schollmeier, R. (2002). A Definition of Peer-to-Peer Networking for the Classification of Peer-to-Peer Architectures and Applications. In: Proceedings of the First International Conference on Peer-toPeer Computing (P2P'01). Linkoping, Sweden, 2001 August 27-29.

Seedorf, P \& Burger, E. (2009). RFC 5693: Application-Layer Traffic Optimization (ALTO) Problem Statement. Retrieved from tools.ietf.org/rfc/rfc5693.txt.

Sjöman, A. (2010). About Voddler. Retrieved from http://voddlertalk.voddler.com/om/

Sourlas, V., Flegkas, P., Paschos, G., Katsaros, D. \& Tassiulas, L. (2011). Storage planning and replica assignment in content-centric publish/subscribe networks. The International Journal of Computer and Telecommunications Networking, 55(18), pp. 4021-4032.

Stabell, C. B. \& Fjeldstad, Ø. D. (1998). Configuring value for competitive advantage: on chains, shops, and networks. Strategic Management Journal, 19(5), pp. 413-437.

Tang, X. \& Chanson, S.T. (2002). Coordinated en-route web caching. IEEE Transactions on Computers, 51(6), pp. 595-607.

Triukose, S., Wen, Z. \& Rabinovich, M. (2011). Measuring a commercial content delivery network. In: Proceedings of the 20th international conference on World wide web. pp. 467-476.

Trossen, D. \& Kostopoulos, A. (2012). Techno-Economic Aspects of Information-Centric Networking. Journal of Information Policy, 2, pp. 26-50.

Vakali, A. \& Pallis, G. (2003). Content delivery networks: status and trends. IEEE Internet Computing, 7(6), pp. 68-74. 
Van der Veen, P. (2011). Orange and Cogent fight over traffic management before French competition watchdog. Retrieved from http:/www.futureofcopyright.com/home/blog-post/2011/09/08/orangeand-cogent-fight-over-traffic-management-before-french-competition-watchdog.html.

Voddler. (2013). Voddler Player Settings. Retrieved from http://www.voddler.com/en/help/topic/2774480800491679958/.

Xu, J., Li, B. \& Lee, D. (2002). Placement problems for transparent data replication proxy services. IEEE Journal on Areas in Communications, 20(7), pp. 1383-1398.

Zhang, L., Estrin, D., Burke, J., Jacobson, V., Thornton, J., Smetters, D. K. .. Yeh, E. (2010). PARC technical report ndn-0001: named data networking (NDN) Project. (Updated 2010 October 31) Retrieved from www.named-data.net/techreport/TR001ndn-proj.pdf.

Zhang, N. (2010). Internet content delivery as a two-sided market. Master's Thesis, Department of Communications and Networking, Aalto University School of Science and Technology.

Zhao, H. (2008). Emerging business models of the mobile internet market. M.Sc. Thesis. Department of Communications and Networking, Helsinki University of Technology.

Zink, M., Suh, K., Gu, Y. \& Kurose, J. (2009). Characteristics of YouTube network traffic at a campus network - Measurements, models, and implications. Computer Networks, 53(4), pp. 501-514. 